\title{
Le Mexique à l'ordre du jour. Les paysans indiens du Chiapas : une question à reconnaître dans l'imagerie évoquée par le climat du nouvel ordre mondial
}

\section{Carlos Aguilar}

\section{Numéro 22, 1994}

URI : https://id.erudit.org/iderudit/1002212ar

DOI : https://doi.org/10.7202/1002212ar

Aller au sommaire du numéro

\section{Éditeur(s)}

Département de sociologie - Université du Québec à Montréal

ISSN

0831-1048 (imprimé)

1923-5771 (numérique)

Découvrir la revue

Citer cet article

Aguilar, C. (1994). Le Mexique à l'ordre du jour. Les paysans indiens du Chiapas : une question à reconnaître dans l'imagerie évoquée par le climat du nouvel ordre mondial. Cahiers de recherche sociologique, (22), 113-133.

https://doi.org/10.7202/1002212ar

\section{Résumé de l'article}

Dans cet article, je propose d'explorer la question concernant la crise politique et sociale déclenchée au Mexique par le soulèvement inattendu des paysans indiens de l'Armée zapatiste du Chiapas. En exposant une autre image du Mexique dans un moment crucial de son histoire, l'Armée zapatiste réactualise les contrastes et les contradictions qui caractérisent ce pays. En alternant et en nuançant les questions de la représentation et des faits historico-politiques soulevés par cette crise, il s'agit d'ouvrir d'autres espaces de discussion en replaçant de telles questions dans un contexte plus large. Le but ultime de cet article est donc de cerner de nouveaux espaces de lisibilité pour reconnaître la question posée par les paysans indiens du Chiapas malgré le climat peu réceptif dégagé par le nouvel ordre mondial. 


\section{Le Mexique à l'ordre du jour. Les paysans indiens du Chiapas: une question à reconnaître dans l'imagerie évoquée par le climat du nouvel ordre mondial}

Hors thème

Carlos AGUILAR

Les Indiens, misérables paysans aux traditions archaïques et conservatrices, isolés par leur inertie des grands courants du Progrès, ou au contraire farouches défenseurs, contre les vents et marées du Colonialisme Blanc, de civilisations incomparables, d'une ethnicité qui porte en germe la rédemption d'un continent.

Christian Deverre, Indiens ou paysans.

Il est impossible de comprendre le mouvement révolutionnaire d'Amérique latine aujourd'hui... sans avoir étudié l'exemple d'Emiliano Zapata.

John Womack, Emiliano Zapata.

\section{Une question à explorer}

La question que je propose d'explorer dans cet article concerne la crise politique et sociale que la rébellion des paysans indiens du Chiapas a déclenchée au Mexique le ler janvier dernier. En se rebellant d'une façon inattendue contre l'autorité (jugée illégitime) du gouvernement mexicain, l'Armée zapatiste a soulevé de vieilles querelles politiques et posé, par conséquent, de nouvelles questions politiques dans les contextes national et mondial.

Considérée principalement à la lumière du contexte de la problématique mexicaine, la crise du Chiapas expose et réactualise le problème des paysans indiens dans un moment crucial de l'histoire mexicaine. Ainsi que la crise du Chiapas le laisse entrevoir, ce moment crucial s'articule autour du programme de modernisation mis progressivement en œuvre par le gouvernement mexicain depuis 1988. D'après la réaction de l'Armée zapatiste, un tel programme de 
modernisation suppose 1 'accomplissement du «génocide ${ }^{1} »$ que les paysans indiens subissent depuis qu'ils ont été «découverts» par la civilisation du Vieux Monde. Selon cette perspective, les paysans indiens du Chiapas se voient sacrifiés définitivement au nom d'une telle civilisation, selon ses modalités sociales et culturelles actuelles.

La réalité sociale du Mexique actuel, un pays qualifié de pays «en transition» (c'est-à-dire qui est en train de passer de façon automatique du sous-développement au développement, d'après le vieux schéma du progrès occidental), mais situé malgré lui dans la périphérie des blocs économiques, fait paraitre sombre l'avenir des paysans indiens en tant que classe sociale sui generis et en tant que peuple de culture différente. Le processus de privatisation qu'impose la modernisation du capitalisme mexicain finira par les chasser définitivement de leurs terres. Ils seront donc obligés de continuer à émigrer vers les villes de Mexico ou du nord du Mexique et viendront élargir ce réservoir de main-d'œuvre bon marché créé par les grands blocs économiques en alliance avec une bourgeoisie mexicaine désormais modernisée. À défaut d'une autre possibilité, ils émigreront sûrement vers les États-Unis et le Canada. Prisonniers de circonstances dues à la déruralisationurbanisation qui caractérise une société comme celle du Mexique, phénomène accéléré et exacerbé par la vague de modernisation économique, les paysans indiens continueront à sombrer dans la marginalisation la plus totale. Mexicanisés, américanisés ou canadianisés, ils finiront par être rejetés dans les marges du monde de la consommation de ces pays.

Avec d'autres problèmes, tels ceux de la démocratisation, du projet de nation, de la légitimité démocratique d'un régime à parti unique, des problèmes liés au processus de déruralisation-urbanisation ont été soulevés par la réaction radicale ${ }^{2}$ des paysans indiens du Chiapas. Il s'agit cependant de problèmes locaux qui se trouvent insérés dans un contexte global caractérisé comme nouvel ordre mondial ${ }^{3}$ dans lequel l'impératif référentiel est désormais l'économique. En effet, sur le plan national, ces problèmes sont tombés sous l'emprise du programme de modernisation lancé par le gouvernement mexicain. Mais, en même temps, ce programme de modernisation se trouve étroitement lié au projet de modernisation à l'échelle mondiale déclenché par le triomphe de «l'idée occidentale», pour emprunter la notion à Francis Fukuyama ${ }^{4}$ à qui je me référerai d'une façon particulière tout au long de cet article.

1 Propos de l'Armée zapatiste cité dans La Presse, Montréal, mardi 4 janvier 1994.

2 En fait, tel qu'énoncé dans leur communiqué de presse du 3 janvier 1994, il s'agit formellement d'une déclaration de guerre (c'est-à-dire comprise comme étant assujettie aux lois de la convention de Genève) contre le gouvernement mexicain, considéré comme démocratiquement illégitime.

3 Pour un aperçu historique d'un tel ordre, voir M. Lefebvre et D. Rotenberg, La genèse du nouvel ordre mondial. De l'invasion de l'Afghanistan à l'effondrement du communisme, Paris, Ellipses, 1992.

4 Voir F. Fukuyama, «La fin de l'histoire?», Commentaire, no 47, été 1989, p. 458. 
L'idée de «la fin de l'histoire» de Francis Fukuyama constitue, sur le plan de la politique pragmatique, un exemple saisissant qui permet de mieux comprendre les enjeux de la modernisation et dudit nouvel ordre mondial. La thèse de Fukuyama est simple: le triomphe (sur l'idéologie communiste) de la démocratie libérale occidentale est «ultime ${ }^{5}$ ». C'est-à-dire qu'il ne s'agit pas seulement de la fin de la guerre froide ou d'une phase particulière de l'après-guerre, mais de «la fin de l'histoire: le point final de l'évolution idéologique de l'humanité et l'universalisation de la démocratie libérale occidentale comme forme finale de gouvernement humain ${ }^{6}$ ».

Plus que la thèse elle-même, ce qui apparaît pertinent chez Fukuyama consiste dans le fait qu'il ne semble pas vraiment croire en une telle thèse et les raisons possibles de son incrédulité. En effet, au début de son essai, Fukuyama constate sur le plan du concret que «la victoire du libéralisme s'est d'abord produite dans le domaine des idées et des consciences et elle est encore incomplète dans le monde réel ${ }^{7}$ ». Cette constatation est suivie d'un sentiment de «nostalgie» et de pessimisme évident dans les dernières lignes de son essai. Les points de suspension de la fin de son analyse expriment d'une sorte d'incomplétude et d'ambivalence envers le triomphe ultime de «l'idée occidentale». Même si Fukuyama reconnaît que «la civilisation qui s'est créée en Europe après 1945» était inévitable, il «éprouve les sentiments les plus ambivalents» à son égard ${ }^{8}$.

Quelle est cette civilisation? Qu'est-ce qui la distingue? Le discours pragmatique de Fukuyama écarte tout ésotérisme philosophique. Le sens de la «démocratie libérale occidentale» dont il parle renvoie en fait à cette civilisation, c'est-à-dire à la «culture de consommation» inventée avant tout par les États-Unis après la Deuxième Guerre mondiale ${ }^{9}$. Pour Fukuyama, le libéralisme politique a suivi le libéralisme économique (les cas de l'Asie et de l'ex-URSS par exemple). Cela signifie que le sens de «démocratie libérale», sens politique par définition, porte en réalité sur l'économique, lequel a réalisé la victoire de cette démocratie. Sur le plan du concret, l'économique est rationalisé en termes de consommation. Dans le cas de l'Asie, l'exemple idéal de Fukuyama, «le désir d'accéder à la société de consommation [...] a joué un rôle crucial dans l'expansion du libéralisme économique $[\ldots]$ et, par là, dans la promotion du libéralisme politique ${ }^{10}$ ». Ainsi, d'après la lecture de Fukuyama, et en dépit de l'idéalité historique que la notion de démocratie porte en elle, la raison d'être de la démocratie occidentale en tant que civilisation repose sur l'économique et son but ultime est celui de «la création

\footnotetext{
5 Ibid.

6 Ibid.

7 Ibid.

8 Ibid., p. 469

9 Ibid., p. 462 .

10 Ibid.
} 
d'une culture de consommation véritablement universelle», une telle culture étant «devenue à la fois un symbole et un support de l'État homogène universel ${ }^{11}$ ».

Comme Fukuyama le laisse entendre à la fin de son essai, son analyse d'orientation hégélienne, basée sur sa lecture de Kojève, se trouve limitée par deux contraintes. D'une part, il s'agit d'une contrainte qui s'étend vers le passé historique: «la grande masse du Tiers-Monde», laquelle «demeure profondément embourbée dans l'histoire» et qui «restera un terrain de conflit pendant de longues années ${ }^{12} \gg$. Pour Fukuyama, «les guerres de libération nationale [parmi d'autres conflits] continueront de représenter un chapitre important de l'ordre du jour international ${ }^{13}$ ». Dans ce nouvel ordre mondial,

[...] le monde serait alors divisé en deux parties: l'une qui serait historique, l'autre qui serait post-historique. Un conflit entre des États encore situés dans l'histoire, ou entre ces derniers et ceux qui se situent à la fin de l'histoire, serait encore possible ${ }^{14}$.

D'autre part, il s'agit d'une contrainte qui s'étend vers l'avenir. C'est la crainte qu'éprouve Fukuyama devant les «siècles d'ennui qui nous attendent après la fin de l'histoire ${ }^{15}$ », c'est-à-dire dans un monde homogène où régnera la «culture de consommation véritablement universelle».

Si on élargit le contexte mexicain en s'appuyant sur la lecture de Fukuyama, la rébellion des paysans indiens du Chiapas devient moins méconnaissable, car elle se trouve bien insérée dans le contexte du nouvel ordre mondial. En ce sens, comme c'est le cas en ce qui concerne sa lisibilité dans le contexte mexicain, la crise du Chiapas pose la question de la possibilité d'autres outils de lecture qui permettent de resituer une problématique caractéristique du monde «historique» (Tiers-Monde) dans des espaces politiques et culturels du monde «post-historique» (premier monde). Par conséquent, la question que pose la crise du Chiapas, question que cherche à circonscrire cet article, est la suivante: dans quelle mesure un événement politique comme celui du Chiapas peut-il être compréhensible dans un monde où les espaces de lecture sont déterminés désormais par l'économique?

En émergeant soudainement de la marginalité totale qui les caractérise, dans laquelle ils étaient représentés par des standards culturels dominants, les paysans indiens du Chiapas cherchent à se représenter eux-mêmes à partir des référents historiques et des héritages culturels qui leur sont propres. Ils amènent avec eux un autre monde, le monde de l' «histoire» dont parle Francis Fukuyama, un monde pré-moderne ou pré-industriel qui était resté caché derrière la façade d'une

11 Ibid.

12 Ibid., p. 466.

13 Ibid., p. 469. (C'est moi qui souligne.)

14 Ibid.

15 Ibid. 
modernisation à caractère impopulaire entreprise par le gouvernement mexicain. Par la nature de leur dissidence et de leurs revendications, ils évoquent d'autres mondes qu'on croyait désormais révolus. D'une part, le monde de la révolution mexicaine, c'est-à-dire celui de l'Armée libératrice du Sud commandée par Emiliano Zapata. D'autre part, le Tiers-Monde, le monde de la décolonisation et des luttes de libération nationale de la période 1950-1970. Ce monde que Fukuyama décrit comme «historique» réapparaît pourtant dans un moment où le premier monde songeait déjà à «la fin de l'histoire», au monde «post-historique» ou post-industriel, post-moderne, post-capitaliste, pour faire allusion aux différents «posties» dont parle Richard Rorty ${ }^{16}$.

Quelle étiquette ou cliché idéologique ou civilisateur faut-il dépoussiérer pour lire cette dislocation contextuelle (pré/post)? Faut-il se demander dans quelle mesure le sous-développement attribué aux paysans indiens est intrinsèque? S'agit-il de l'anachronisme typique d'un monde retardé, resté prisonnier du politique auquel fait allusion Fukuyama, c'est-à-dire un monde «historique» qui doit lutter encore «pour [sa] reconnaissance» par rapport au monde «posthistorique» du «calcul économique» et des «consommateurs sophistiqués ${ }^{17}$ »?

Compte tenu de l'enjeu politique et culturel de cette dislocation contextuelle, ces questions supposent l'exploration des espaces de lisibilité à partir desquels la question politique soulevée par des paysans indiens du Chiapas peut être reconnue dans un monde où règne désormais l'économique. Il s'agit alors, à partir de cette introduction, de repérer dans un premier temps les possibilités de lecture de la crise du Chiapas selon d'autres espaces de discussion qui peuvent être ouverts dans le contexte de la problématique mexicaine, principalement. Dans un deuxième temps, il s'agit de considérer cette problématique à la lumière du contexte de désordre désigné par ledit nouvel ordre mondial, malgré le climat politique peu réceptif qui le caractérise.

\section{La guerre des images}

Parce que l'image constitue avec l'écrit l'un des outils majeurs de la culture européenne, la gigantesque entreprise d'occidentalisation qui s'abattit sur le continent américain assuma - en partie du moins - la forme d'une guerre des images qui se perpétua pendant des siècles et dont rien n'indique qu'elle soit close aujourd'hui.

Serge Gruzinski ${ }^{18}$

16 Cité par J. Rajchman, «Habermas's Complaint», New German Critique, no 45, automme 1988, p. 167.

17 F. Fukuyama, article cité, p. 469.

18 S. Gruzinski, La guerre des images, de Christophe Colomb à «Blade Runner» (14922019), Paris, Fayard, 1990, p. 13. (C'est moi qui souligne.) 
Le monde des paysans indiens du Chiapas semble être un monde historiquement brouillé, imagé et méconnu. En dépit de la pluralité des registres culturels qui le caractérisent, comme le souligne Serge Gruzinski ${ }^{19}$, il s'agit d'un monde systématiquement dévalorisé par une telle entreprise d'occidentalisation. Les intérêts de l'habitude historiographique, politique, scientifique ou touristique tendent à représenter et à imaginer le monde des paysans indiens en le distordant. Comme d'autres cultures dominées, le monde des paysans indiens, taxé par exemple d'indianisme par la pratique discursive standardisée et spécialisée, est un monde qui tend à être réduit au modèle culturel imposé par l'Occident.

Edward Saïd cite Karl Marx au sujet de ceux qui «ne peuvent se représenter eux-mêmes; [mais qui] doivent être représentés ${ }^{20}{ }_{\gg}$. Dans ce propos Marx faisait sûrement référence aux travailleurs (au prolétariat) en tant que classe sociale dépourvue des moyens matériels et culturels pour se représenter elle-même. Autrement dit, pour se représenter elle-même, une «autre» culture, une culture dominée et marginalisée par le processus d'occidentalisation (de droite ou de gauche), doit acquérir les moyens politiques et culturels (donc matériels). C'est ce qu'a réussi à faire jusqu'à maintenant l'Armée zapatiste sur le plan politique: acquérir des moyens politiques pour être en mesure d'ouvrir d'autres espaces de discussion qui permettront de resituer tant la problématique paysanne indienne que la problématique mexicaine dans le contexte de désordre provoqué par le susdit nouvel ordre mondial. En effet, la représentation que les paysans indiens du Chiapas (et ceux d'autres régions du Mexique qui les rejoignent politiquement) ont d'eux-mêmes est acquise présentement par leur participation sociale et historique.

La problématique paysanne indienne suppose une ambivalence d'ordre historique soulignée explicitement tout au long de cet article par la référence indistincte aux paysans et aux Indiens. En qualité de paysans, ils forment une classe sociale sui generis bien marquée historiquement lors de la révolution mexicaine et, comme Indiens, ils constituent un peuple de culture différente, syncrétique. Il s'agit alors d'une ambivalence à caractère socio-historique qui tend à être méconnue par la domination des modèles culturels (français, britannique ou américain) standardisés lors de l'entreprise d'occidentalisation dont parle Serge Gruzinski ${ }^{21}$.

Pour mieux montrer l'importance d'une telle ambivalence, il importe cependant de signaler la représentation historique qui est à l'origine de la méconnaissance du problème paysan-indien, compte tenu que l'Armée zapatiste elle-même situe historiquement sa rébellion dans une tradition de luttes de résistance qui dure depuis la «découverte» du Nouveau Monde. Dès la première

19 S. Gruzinski, La colonisation de l'imaginaire. Sociétés indigènes et occidentalisation dans le Mexique espagnol, XVIe-XVIIIe siècle, Paris, Gallimard, 1988. Voir notamment l'introduction.

20 E. Saï, L'orientalisme. L'Orient créé par l'Occident, Paris, Seuil, 1980, p. 11.

21 S. Gruzinski, La guerre des images, ouvrage cité. 
phrase de son communiqué du 3 janvier (1994), l'Armée zapatiste déclare être «le produit de 500 ans de lutte». En effet, dès sa «découverte» par la civilisation occidentale, le monde des Indiens commence à être brouillé. Selon Beatriz Pastor, c'est dès ce moment que commence un processus de méconnaissance, d'instrumentation et de destruction de la nouvelle réalité américaine qui s'est prolongé jusqu'à aujourd'hui ${ }^{22}$.

Considérant l'intérêt pour la connaissance que la civilisation européenne éprouvait déjà à ce moment, on aurait espéré que, à la suite de leur «découverte», les conquistadores entreprennent un «processus de connaissance» de la nouvelle réalité. Mais ce qui se produisit fut, au contraire, la réduction de celle-ci au modèle idéologique ou de représentation qui caractérisait déjà à ce moment l'univers de la culture européenne. D'après l'étude de Beatriz Pastor, un tel modèle s'articule autour de deux éléments qui se trouvent historiquement interreliés: d'une part, l'intérêt commercial, plus ou moins explicite, à partir duquel on concevait les nouvelles terres découvertes et ses richesses comme un butin légitime à saccager ${ }^{23}$ et, d'autre part, la justification religieuse de l'aspect commercial d'une telle «découverte».

Conçue comme une mission à caractère divin, la «découverte» avait pour idéal suprême l'expansion de l'empire de la chrétienté; une telle mission était semble-til motivée par la nécessité qu'éprouvaient les indigènes (les infidèles) de s'intégrer à l'univers chrétien. Ce modèle de représentation dominant a servi comme mécanisme de réduction, de déformation et d'invention de la nouvelle réalité en dépit de sa diversité ${ }^{4}$. Ainsi, à l'encontre des idées reçues depuis lors, il ne s'agit pas de la «découverte» d'une nouvelle réalité, mais plutôt «de l'idée» selon laquelle cette réalité avait été découverte ${ }^{25}$.

Aujourd'hui, en réutilisant les mécanismes d'invention d'images du monde médiatique, les paysans indiens du Chiapas se rebellent contre ce modèle de représentation dominant ${ }^{26}$. En projetant d'une façon inattendue une autre image du

22 Voir B. Pastor, Discurso narrativo de la Conquista de América, La Habana, Cuba, Casa de las Américas, 1983, p. 17.

23 Ibid., p. 37-42. Beatriz Pastor fait référence ici à la correspondance (1480) entre Paolo Toscanelli et Christophe Colomb.

24 Ibid. Le sens historique d'un tel modèle se trouve étroitement lié, selon l'auteur, à l'esprit des Croisades.

25 Je fais référence à l'étude de E. O'Gorman, La invención de América, Mexico, Fondo de Cultura Económica, 1984, p. 10 et suiv.

26 L'exemple le plus surprenant est celui de l'archi-célèbre sous-commandant «Marcos». A force de le représenter, la machine médiatique a fini par recréer un cliché à travers lequel l'Armée zapatiste communique ses messages: "Je suis un mythe génial..."», reconnaît le sous-commandant «Marcos» lui-même (B. de la Grange, Le Devoir, Montréal, 23 février 1994, p. A1). Par ailleurs, en essayant de «critiquer» un tel mythe, ce journaliste se voit glisser dans le jeu des images créées par la machine médiatique. 
Mexique dans l'opinion publique mexicaine et mondiale, l'Armée zapatiste fait la lumière sur une réalité qui s'avère plus complexe que celle projetée par l'image habituelle. Les problèmes qui caractérisent un pays comme le Mexique, pays que le gouvernement mexicain essaie d'intégrer de façon draconienne audit premier monde, viennent d'être exposés dans le cadre d'un nouvel espace politique qui fait perdre son innocence à l'image généralement répandue d'un Mexique folklorique et progressiste. L'image stéréotypée créée à la fois par les médias de masse et par les intérêts nationaux et internationaux de l'industrie touristique et de la politique étrangère du gouvernement mexicain commence à s'effriter.

Indépendamment des interprétations auxquelles la crise du Chiapas a donné et donnera lieu dans les cercles d'opinion à caractère gouvernemental, journalistique et intellectuel du Mexique (cet univers générateur d'opinions faisant autorité), indépendamment aussi de la durée et de l'issue (politique ou militaire) que connaîtra cette crise, le Mexique ne devrait plus continuer à être perçu comme auparavant. «Quelque chose vient d'éclater dans nos consciences», remarque l'écrivain et journaliste mexicaine Elena Poniatowska à la suite de la rébellion de l'Armée zapatiste. «Du jour au lendemain tout a changé. En 1994, nous nous sommes réveillés dans un pays différent ${ }^{27} . »$

Les vieux mythes qui hantaient l'imaginaire social des Mexicains s'effacent à mesure que la crise du Chiapas met en évidence les enjeux sociaux et politiques des problèmes soulevés: révolution mexicaine, grande famille mexicaine, stabilité sociale, solidarité, modernisation. Cette imagerie suscitée par le discours du gouvernement mexicain est en train de voler en éclats. L'image de la révolution mexicaine, par exemple, si elle devait avoir encore un sens quelconque pour l'imaginaire social des Mexicains, est usée; elle a été dévaluée par la surutilisation qu'en a fait le Parti révolutionnaire institutionnel (PRI) - et par l'historiographie officielle - durant les soixante-quatre ans qu'il a passés au pouvoir. Du point de vue constitutionnel, et en tant que référent historique donnant du sens à l'idée de nation, la révolution mexicaine devrait être porteuse d'un projet de société qui légitimerait sa référence. Dans ce projet virtuel de société, les paysans indiens devraient avoir une place dans une «nation mexicaine» pourtant reconnue comme plurielle et diverse, comme il est dit par exemple dans le document distribué au Canada par le gouvernement mexicain lors de la promotion de l'ALÉNA ${ }^{28}$.

D'après ce document, au norn du «nationalisme progressiste», le président Salinas de Gortari s'est donné comme tâche de moderniser la vieille image de la révolution mexicaine. Mis à part la rhétorique modernisatrice du président mexicain, une telle tâche consiste en fait dans le recyclage purement lexical de la vieille image de la révolution mexicaine pour l'adapter aux exigences de la vague

27 E. Poniatowska, «Chiapas o la desesperación», La Jornada, Mexico, 9 janvier 1994, p. 12.

28 Voir La chronique du Mexique, Présidence du Mexique, Direction générale des services de presse, octobre 1990, p. 77. 
néolibérale triomphante. Sous la rubrique «La réforme de l'État», l'auteur de $L a$ chronique du Mexique cite amplement le discours du président Salinas de Gortari:

Rien n'est plus étranger aux idéaux de la Révolution mexicaine que l'immobilisme face à tout ce que nous devons faire. La modernisation nous donne le moyen d'atteindre réellement les objectifs de la Révolution. Nous changeons parce que nous voulons faire de la Révolution une réalitê29.

Laissons de côté ce «nous» insistant qui se veut représentatif de la société mexicaine pour bien légitimer le propos du discours du président. Il importe plutôt de souligner la notion de «réalité» par laquelle Salinas de Gortari laisse entendre que, jusqu'au moment de la modernisation qu'il a entreprise en 1988, la révolution mexicaine ne semble pas avoir été une réalité. Le discours de Salinas de Gortari surprend, car c'est précisément en faisant appel à la révolution mexicaine que son parti a légitimé politiquement sa présence au pouvoir depuis 1929. Mais que sous-entend Salinas de Gortari lorsqu'il fait allusion à «la modernisation»? Il veut probablement rappeler que le contexte socio-politique mondial dans lequel évolue le Mexique depuis les dernières années a changé. C'est-à-dire que le processus d'occidentalisation dynamisé par la mondialisation du capitalisme a atteint une nouvelle phase, laquelle exige une réforme en profondeur du politique considéré désormais comme secondaire, voire presque anachronique. Il ne faut pas oublier par ailleurs qu'à ce moment (1990), le slogan du Cambio («changement») promu officiellement par le gouvernement mexicain avait pour but de sensibiliser l'imaginaire social des Mexicains à la politique modernisatrice.

On se trouve donc devant le grand tournant, devant une nouvelle situation sociale et politique qui requiert un nouveau champ référentiel à partir duquel seront dorénavant standardisés les nouveaux espaces sémantiques qui soutiennent le discours politique du gouvernement mexicain: la modernisation. La vieille notion de nationalisme sera recyclée; en l'orientant vers l'avenir (plutôt que vers le passé), elle deviendra ce que Salinas de Gortari appelle «nationalisme progressiste». Le recyclage de la vieille formule politique de «Révolution» sera réalisé au nom d'un tel nationalisme. Mais Salinas de Gortari ne vise pas à modifier les mots sur le plan purement lexical, sens dépourvu des enjeux politiques et des implications historiques; ce qu'il cherche, c'est de moderniser un telle signification sur le plan historique. Dès lors, le président mexicain fera plutôt appel aux «idéaux» et aux «objectifs» de la révolution mexicaine. C'est en ce qui concerne cet aspect que les modernisations lexicales réalisées par Salinas de Gortari créent des tensions. En effet, vouloir moderniser les idéaux et les objectifs de la révolution mexicaine par une nouvelle rhétorique sans aborder concrètement les problèmes criants du Mexique en accord avec l'esprit (social et populaire) de la Constitution de 1917, constitue une entreprise vouée à l'échec.

29 Ibid., p. 51. (C'est moi qui souligne.) 
À travers des discours où s'entrecroisent les mêmes référents historiques légitimant leurs positions, l'Armée zapatiste et le gouvernement de Salinas de Gortari font partie de cette guerre des images dont parle Serge Gruzinski ${ }^{30}$. Loin de ressembler à un simple jeu d'illusions, de telles images tendent à évoquer plutôt les enjeux politiques et historiques des vieux problèmes soulevés.

En effet, la visée de la modernisation politique de Salinas (réalisée en fonction de l'économique) est le renouvellement d'une idée de révolution mexicaine considérée désormais comme obsolète. Dans son discours, Salinas parle d'une «révolution sans mythes ${ }^{31}$ ». Engendrés par le «gigantisme de l'État», ces mythes découlent, selon Salinas, «d'un vieux concept de révolution». En se démarquant ainsi d'un concept de révolution que Salinas juge révolu, le but politique du président mexicain consiste à justifier à long terme la modernisation de l'État. On constate alors, d'une part, que la «grande tâche» que Salinas s'assigne consiste à «consolider la réforme de la révolution» et, d'autre part, que l'idéologie qui oriente ce grand virage est celle du «libéralisme social, synthèse de la réforme et de la révolution». Salinas de Gortari fait référence aux deux moments constitutifs du Mexique en tant que nation: à la Constitution de 1857 issue de la guerre dite «de réforme» (1857-1867), dans laquelle le triomphe de l'idéologie libérale a ouvert le chemin au Mexique capitaliste ${ }^{32}$, et à la Constitution de 1917 issue de la révolution mexicaine (1910-1920). La lutte de l'armée des paysans indiens commandée par Emiliano Zapata (ainsi que par Francisco Villa) a donné une orientation populaire, donc sociale, à cette dernière constitution, sur laquelle est encore fondée la légitimité de la nation mexicaine.

La modernisation politique proposée par le président mexicain est mise en œuvre au nom de «Juárez [président mexicain d'origine indienne lors de la période dite de la Réforme - 1861] et de Zapata ${ }^{33}$ ». L'admiration que Salinas de Gortari porte à l'image d'Emiliano Zapata est bien connue au Mexique. En tant qu'image aseptisée, vidée de son sens historique, le nom de Zapata dans le discours de Salinas détient plutôt une fonction immunisante. Mais dès que l'autre image de Zapata glisse de la simple rhétorique vers son sens historique, le discours de Salinas de Gortari s'annonce paradoxalement prémonitoire envers l'éventuelle crise du Chiapas. En effet, pour Salinas (en dépit du grand virage vers l'économique?), «dans le pays, il y aura toujours des luttes pour la justice sociale tant et aussi

30 S. Gruzinski, La guerre des images, ouvrage cité.

31 Voir l'article de S. Loya, «Salinas preconizaba una "revolución sin mitos"...», Proceso, Mexico, no 897, 10 janvier 1994, p. 21. Les citations qui suivent dans ce paragraphe sont tirées de cet article.

32 Voir A. Gilly, La revolución interrumpida. México, 1910-1920: una guerra campesina por la tierra y el poder, Mexico, El Caballito, 1971. Voir notamment «El desarrollo capitalista».

33 S. Loya, article cité. 
longtemps que dans le cœur des Mexicains battra la mémoire et l'exemple d'Emiliano Zapata ${ }^{34}$ ».

À l'encontre du discours du président Salinas de Gortari, l'Armée zapatiste ne fait pas référence à la révolution mexicaine en la nommant explicitement. La perspective politique générale dans laquelle l'Armée zapatiste situe sa rébellion est celle de la lutte de résistance des paysans indiens encadrée historiquement selon les phases constitutives de la nation mexicaine: la guerre d'Indépendance contre les empires espagnol, français et américain respectivement, ainsi que la lutte contre la dictature de Porfirio Díaz (1877-1910), laquelle a débouché sur l'événement historique qu'on appelle révolution mexicaine ${ }^{35}$.

Le référent historique principal de l'Armée zapatiste, celui qui inspire la rébellion et qui lui donne un sens, c'est l'héritage d'Emiliano Zapata: «Le programme idéologique et politique de l'Armée zapatiste de Libération nationale s'est inspiré de l'esprit libérateur du chef révolutionnaire du Sud, le général Emiliano Zapata ${ }^{36} \gg$. Leur revendication politique la plus fondamentale est formulée par rapport à l'article 39 de la Constitution de 1917, un des articles les plus importants de cette charte qui découle, en ce qui concerne son orientation populaire et sociale, de la tradition zapatiste:

[...] la souveraineté nationale réside essentiellement [...] dans le peuple. Tout pouvoir public émane $d u$ peuple et il est institué pour son bénéfice. Le peuple a, en tout temps, le droit inaliénable d'altérer ou de modifier la forme de son gouvernement ${ }^{37}$.

C'est en s'appuyant sur cet article que l'Armée zapatiste déclare la guerre au gouvernement de Salinas de Gortari, en ce sens qu'il est considéré comme illégitime à la suite des accusations de fraude lors des élections en 1988. Traduite en termes politiques (espace privilégié où l'Armée zapatiste trouve sa raison d'être ${ }^{38}$ ), une telle déclaration de guerre signifie «la démission du gouvernement fédéral et la formation d'un nouveau gouvernement de transition qui déclenchera des élections libres et démocratiques en août $1994^{39}$ ». On remarque donc que c'est par

34 Ibid.

35 Voir le communiqué du 3 janvier 1994. Voir aussi G. Correa et I. Ramírez, «A Emiliano Zapata y su Plan de Ayala atribuye el Ejército Zapatista de Liberación Nacional su inspiración ideológica», Proceso, no 897, 10 janvier 1994.

36 G. Correa et I. Ramérez, article cité.

37 Ibid. (C'est moi qui souligne.)

38 Voir «Revolución electoral o el país se levantará, advierte Marcos», entrevue accordée par le sous-commandant «Marcos» au quotidien La Jornada, Mexico, mardi 8 février 1994.

39 Voir E. Maza, «El comandante Marcos, al periódico "L'Unità"...» (entrevue), Proceso, Mexico, no 897, 10 janvier 1994. Par ailleurs, l'Armée zapatiste a mis pour le moment de côté ses demandes en attendant les résultats des négociations entreprises 
le biais des mots «liberté et démocratie» que l'Armée zapatiste a réussi à rejoindre les paysans indiens d'autres régions du Mexique et les couches populaires en général. Ces termes sont spécialement significatifs ces jours-ci au Mexique.

Ce qui est en jeu dans la situation esquissée jusqu'ici, c'est le sens même de modernisation, sens qui soulève les questions du politique et de l'économique. On sait avec la lecture de Fukuyama qu'après l'effondrement dans le sillage du communisme, donc du triomphe de «l'idée occidentale» et de l'interprétation de la nouvelle situation qui en découle comme s'agissant d'un nouvel ordre mondial, le politique est devenu désormais démodé face à l'économique. Ainsi, ayant comme nouveau standard référentiel l'économique, la réforme politique que le gouvernement de Salinas a amorcée a été réalisée en fonction de la modernisation économique. Il s'agit alors d'une modernisation partielle et impopulaire en ce sens que, dans le contexte d'un pays comme le Mexique, pays qui demeure profondément embourbé «dans l'histoire» (en rappelant la lecture de Fukuyama ${ }^{40}$ ), la société et la nation mexicaines prennent encore leur sens historique en se référant à l'esprit social et populaire émanant de la Constitution de 1917. C'est cette partialité et cette impopularité de la modernisation entreprise par le gouvernement de Salinas de Gortari que la crise du Chiapas vient mettre en évidence.

Le gouvernement mexicain, c'est-à-dire le PRI en tant que parti unique, se trouve devant un dilemme qui présente différents volets. D'une part, pour que l'économique joue pleinement son rôle comme référent déterminant dans le contexte mexicain, donc sans la contrainte du politique, il ne suffit pas de réformer ou de moderniser le lexique de la révolution mexicaine; il faudra plutôt déraciner le référent politique et historique à partir duquel prend son sens la société mexicaine. Il s'agirait ainsi de la modernisation idéale tant souhaitée par les agents locaux et mondiaux du progrès économique. D'autre part, considérant l'état actuel dans lequel se trouve la société mexicaine quant à son sens historique, il apparaît difficile pour le gouvernement mexicain de prétendre déraciner le référent historique sans soulever de sérieux problèmes politiques. La réforme de l'article 27 de la Constitution, qui concerne le droit des paysans indiens à la propriété collective de la terre, est un exemple concret d'un tel dilemme, car c'est précisément cette réforme qui a déclenché la crise du Chiapas. Finalement, il convient de considérer aussi le sort du PRI devant un tel dilemme, compte tenu de sa «particularité historique ${ }^{41}{ }^{1}$.

avec le gouvernement mexicain. Lire Le Devoir, Montréal, mercredi 23 février 1994, p. B5.

40 F. Fukuyama, article cité, p. 466.

41 Je fais allusion à l'analyse que César Velázquez Robles fait du PRI dans la présente conjoncture. Voir «Reforma del partido, reforma del poder», La Marcha, Mexico, no 1, 14 mars 1994 , p. $20-21$. 
Contrairement aux autres partis politiques qui sont apparus pour organiser la prise du pouvoir politique, le PRI est le résultat du pouvoir même qui a émergé pour garantir l'unité des forces sociales qui se sont associées lors de sa formation. Autrement dit, le PRI est né au moment de l'institutionnalisation de la révolution mexicaine; il apparaît donc en même temps que l'État issu d'une telle révolution. Prétendre déraciner le référent historique qui donne leur sens à la société et à la nation mexicaines pour qu'une modernisation économique idéale puisse s'épanouir sans contraintes du politique signifie alors la disparition du PRI lui-même en tant que parti-État. Le contexte global de lisibilité de cette nouvelle situation fortement paradoxale à laquelle fait face le gouvernement mexicain serait celui qu'Adolfo Gilly décrit comme «la réorientation radicale de la politique sociale et économique du gouvernement mexicain ${ }^{42} \gg$.

Le politique et l'économique supposent aussi les questions de l'État et des lois de l'économie du marché (ou le laisser faire économique). En réformant l'État, en le «privatisant», le gouvernement mexicain provoque une situation doublement conflictuelle. D'un côté, l'État ayant été réformé (neutralisé politiquement, tel que le voulaient les intérêts des agents de la modernisation), son pouvoir politique pour réaliser de tels idéaux est relégué aux lois de l'économie du marché, lesquelles, par leur logique interne, n'ont rien de démocratique. D'un autre côté, le renoncement à son rôle de garant politique de ces idéaux, tel que le stipule la Constitution de 1917 , crée un vide et une instabilité politiques. Le problème réside alors dans le caractère non constitutionnel du nouvel agent (implicite) réalisateur des idéaux de la révolution, c'est-à-dire les lois du marché ou le laisser faire économique.

Par suite du changement draconien de l'État en tant qu'agent réalisateur original, la tâche réformatrice de Salinas de Gortari consiste à justifier un tel changement en faisant appel aux vieilles formules politiques évocatrices de la révolution mexicaine. La situation semble être dramatique, comme le montre la crise déclenchée par la rébellion zapatiste. De fait, le vieil État mexicain a été réformé pour répondre aux exigences d'une nouvelle situation (c'est-à-dire d'un nouvel ordre mondial), mais le parti politique de Salinas de Gortari (le PRI) lié à ce vieil État depuis soixante-quatre ans se trouve toujours au pouvoir.

Dans un pays comme le Mexique, caractérisé par un système politique à parti unique et par des problèmes de développement et de dépendance économiques chroniques, une modernisation politique et économique conçue globalement est fondamentale. Le paradoxe politique engendré par la modernisation partielle et impopulaire amorcée par le gouvernement mexicain, dont bénéficie uniquement l'économique (privatisations, investissements, par exemple), devient évident dès qu'on considère une telle modernisation sous l'angle de l'expérience d'autres pays où régnaient des partis uniques, comme c'est le cas des pays de l'Europe de l'Est.

42 A. Gilly, Mexique. Notre chute dans la modernité, traduit de l'espagnol par V. Giraud et Y. Sintomer, Paris, Éditions Syllepse, 1992, p. 5. 
Dans ces pays, l'État a été réformé lors de l'effondrement du communisme mais, à la différence du parti unique mexicain, les partis au pouvoir qui se trouvaient en symbiose avec le vieil État ont connu le même sort. En ce sens, Adam Michnik, réformateur politique polonais assez connu, en faisant référence à la situation politique créée par des partis uniques, soulignait alors que les réformateurs polonais «parviendront à une véritable démocratie plus vite que le Mexique ${ }^{43}$ ».

Pour ce qui est du vide politique issu d'un tel paradoxe, on se rend compte qu'on se trouve dans une sorte de situation limite ou devant un dilemme, comme je l'ai souligné plus haut. On peut faire référence à une telle situation en rappelant à nouveau l'analyse de Fukuyama en ce qui concerne le contraste qui se manifeste entre les conditions pré-modernes ou «historiques» dans lesquelles évoluent des pays comme le Mexique et les conditions post-modernes ou «post-historiques» des pays du premier monde.

Au contraire de la plupart des sociétés du premier monde, où l' «homme économique» engendré par la culture de consommation semble remplir ce vide en remplaçant le politique par l'économique, le cas d'un pays comme le Mexique semble différent. En effet, la culture de consommation étant moins développée, un tel vide politique ne pourra pas être rempli par l'«homme politique» de la société mexicaine selon les conditions établies par la société de consommation. En attendant que la culture de consommation rationalise et homogénéise (perfectionnant du coup le processus d'occidentalisation dont parle Serge Gruzinski ${ }^{44}$ ) idéalement la société mexicaine, l'«homme politique» qui la caractérise tend à remplir un tel vide en valorisant davantage le politique. En exposant les enjeux politiques et historiques de la problématique mexicaine, la crise du Chiapas continue à montrer avec netteté l'ampleur d'un tel dilemme. Adolfo Gilly le décrit ainsi:

La crise du Mexique se concentre dans le royaume de la politique. Elle s'est transformée en une crise de la conscience nationale. Comme en Tchécoslovaquie ou en Allemagne de l'Est, à l'aube des années quatre-vingtdix, le régime politique mexicain est entré de manière irréversible dans la fin d'une époque: son maintien indéfini est incompatible avec la volonté explicite d'une société en mouvement ${ }^{45}$.

En la simplifiant d'après ses effets sociaux, une telle situation met en évidence l'imagerie à laquelle fait appel la modernisation économique entreprise par le gouvernement mexicain. Il s'agit d'une situation dans laquelle les gens qui se trouvent sur les bons échelons de la hiérarchie sociale se voient déjà dans le premier monde, dans le monde «post-historique», tandis que les autres, c'est-à-dire

43 A. Michnik, La deuxième révolution, Paris, Éditions La Découverte, 1990, p. 203.

44 S. Gruzinski La colonisation de l'imaginaire, ouvrage cité.

45 A. Gilly, Mexique. Notre chute dans la modernité, ouvrage cité, p. 133. (C'est moi qui souligne.) Voir notamment «Le dilemme du régime mexicain». 
les paysans indiens et les autres secteurs sociaux les plus marginalisés, se voient, eux, exclus et refoulés davantage dans les conditions «historiques» du monde du politique.

La rébellion des zapatistes tourne autour de cette question. D'après les communiqués et les propos que tient l'Armée zapatiste lors des entrevues, modernisation et démocratisation vont de pair pour elle. En désapprouvant l'ALÉNA (perçu comme le couronnement du programme de modernisation), elle remet en question la légitimité du gouvernement de Salinas de Gortari, légitimité qui a été ternie à la suite d'accusations de fraude portées contre son parti (PRI) par l'opposition lors des élections de $1988^{46}$. Cela soulève la question de la démocratisation, donc le besoin d'espaces politiques nouveaux dans un Mexique politiquement contrôlé.

Le climat de réceptivité créé par les zapatistes chez d'autres paysans indiens (et parmi les couches populaires en général) dans le centre et le sud-est du Mexique, montre que dle nouveaux espaces politiques sont en train de s'ouvrir: «Nous ne sommes pas zapatistes, mais la pauvreté est la même», déclare un leader paysan indien lors de la prise de la mairie du village de Teopisca, dans le sud-est du Mexique. Pour leur part, en revendiquant «les 500 ans de résistance indigène», des paysans indiens de l'État de Guerrero (à l'ouest de Chiapas), ont entamé une marche vers la ville de Mexico «pour appuyer l'Armée zapatiste». Leur message: «Les indigènes, jamais plus citoyens de seconde classe ${ }^{47}$ ».

En contraste avec la modernisation politique entreprise par Salinas de Gortari, laquelle est conçue uniquement en fonction de l'économique, on remarque qu'en soulevant la question de la démocratisation du Mexique, l'Armée zapatiste propose une «révolution électorale ${ }^{48}$ », c'est-à-dire politique. On se trouve donc face à une situation limite qui «se concentre dans le royaume de la politique», selon l'analyse d'Adolfo Gilly 49 . S'il fallait choisir une métaphore pour décrire cette situation limite, ce serait celle d'espace politique. En effet, en dépit de la diversité des points de vue exprimés au Mexique autour de la crise du Chiapas, une opinion traverse la presse écrite mexicaine, à laquelle je me réfère tout au long de cet article: en ouvrant de nouveaux espaces de discussion, les paysans indiens du Chiapas élargissent la perspective de la politique mexicaine.

46 Voir «Crónica de un fraude», vidéo réalisée par C. Mendoza (Canal 6 de julio, Mexico, 1988).

47 En ce qui concerne le village de Teopisca, voir M. S. Serril, «Zapatismo Spreads. Inspired by the rebels of Chiapas, the downtrodden are taking their grievances to the streets», Time, 21 février 1994. En ce qui à trait à la marche de Guerrero, voir Le Devoir, Montréal, mercredi 23 février 1994, p. B5.

48 Voir «Revolución electoral o el país se levantará, advierte Marcos», article cité.

49 A. Gilly, Mexique. Notre chute dans la modernité, ouvrage cité, p. 133. 


\section{Le nouveau désordre mondial}

Le nouvel espace politique que la rébellion des zapatistes vient de créer dans le contexte mexicain permet de faire référence (en le questionnant) au statu quo qui règne dans le champ du politique, et ce dans le contexte mondial, quant à la problématique qui caractérise un pays comme le Mexique. Il s'agit de repenser une telle problématique et d'entrevoir d'autres perspectives du politique qui permettraient d'élargir le sens d'un monde désormais conçu comme définitif par la logique néolibérale triomphante. Il importe également de penser à d'autres espaces de discussion sur les nouvelles vérités (économiques) qui sont en train d'homogénéiser le monde contemporain, c'est-à-dire le nouvel ordre mondial et son programme de modernisation, lequel tend à créer ce que craint Fukuyama: «une culture de consommation véritablement universelle ${ }^{50}$ ».

En dépit de l'imagerie à laquelle se réfère le «nouvel» ordre mondial, il semble que celui-ci soit plutôt le résultat d'une lecture déformatrice des aspects du réel politique et social, du fait d'une interprétation de l'effondrement du communisme en tant que triomphe de «l'idée occidentale». Cela semble paradoxal, car on tend à croire qu'après l'effondrement de l'idéologie communiste, il règne dans le monde une nouvelle ère, celle de «la "fin des idéologies"», selon la lecture de Fukuyama par exemple ${ }^{51}$. Pourtant, cette nouvelle ère témoigne justement d'une lecture idéologique en ce sens que, le spectre du communisme ayant disparu, ce que les agents de la modernisation désirent, c'est de continuer à réaliser le vieux rêve de «l'idée occidentale»: l'occidentalisation de la planète ou, en d'autres mots, la conquête, le contrôle et l'ordonnancement, à l'échelle planétaire, de tous les espaces.

Considérée historiquement en tant que culture ou civilisation dominante, «l'idée occidentale» consiste en fait à civiliser et à occidentaliser les «barbares» à travers des campagnes de modernisation ${ }^{52}$. Une telle occidentalisation ne se limite pas aux «barbares» du Tiers-Monde colonisé, néocolonisé ou impérialisé, comme le voudrait le vieux modèle manichéen, mais elle s'étend aussi aux exclus des sociétés du premier monde. En effet, on tend à décrire le phénomène urbain de marginalisation dans les sociétés du premier monde comme un phénomène de tiersmondialisation (les chômeurs, les sans-abri, par exemple). Historiquement, en ce qui concerne le Tiers-Monde proprement dit, pour les paysans indiens du Mexique, et pour les Indiens du continent américain en général, le rêve de «l'idée occidentale» a été vécu plutôt comme un cauchemar, sous ses modalités colonialiste, néocolonialiste ou impéraliste.

50 F. Fukuyama, article cité, p. 462.

51 Ibid., p. 458.

52 Voir E. Saïd, par exemple Culture and Imperialism, New York, Alfred A. Knopf, 1993. 


\section{No man's land}

À en juger par la dévastation du social provoquée par la modernisation du capitalisme mexicain (à l'origine de la crise du Chiapas), par les «investissements pervers en Amérique latine ${ }^{53}$ », par la «perversion de la démocratie ${ }^{54}$ » qui fait des ravages dans les sociétés du premier monde et par la déstabilisation et l'appauvrissement engendrés par le triomphe «de l'idée occidentale» dans le monde ex-communiste ${ }^{55}$, ledit nouvel ordre mondial (malgré l'imagerie qu'il a créée) n'évoque en réalité rien de nouveau. Derrière la façade de la nouvelle image, nous retrouvons plutôt un nouveau désordre mondial que «l'idée occidentale» prétend ordonner en s'investissant d'une tâche missionnaire. Comme les exemples de la France (par les événements actuels qui évoquent mai 68) et de l'Italie (références de plus en plus explicites à l'ordre prêché par il Duce) l'illustrent, cette situation chaotique génère des réactions et pose des difficultés politiques quant aux solutions à proposer. En ce qui concerne le désordre mexicain, il semble que ce sont les paysans indiens de l'Armée zapatiste qui remettront l'histoire en marche en créant de nouveaux espaces politiques.

Après l'euphorie qu'a suscitée l'effondrement du communisme, dont l'apogée fut la victoire des «forces alliées» lors de la guerre du Golfe ${ }^{56}$, «l'idée occidentale» s'affaiblit peu à peu. Le moment actuel, qu'on le décrive comme post-historique, post-industriel, post-moderne ou post-capitaliste reste historique selon un sens bien précis. Dès l'effondrement du communisme, le spectre de cette idéologie semble s'être évanoui définitivement. Il convient de souligner cependant qu'ayant été créé originellement par les conditions du système communiste, ailleurs, hors du contexte des pays communistes, comme ce fut le cas dans les pays du TiersMonde, ce spectre a été recréé notamment par la propagande idéologique dudit monde libre pendant la période de la guerre froide.

Le communisme vaincu, en esprit et en matière, «l'idée occidentale» semble se trouver seule face à elle-même. La confusion du président Clinton lors de la préparation de sa première mission en Europe nous donne un aperçu du point de vue pragmatique de ce nouveau moment d'imprécision (de crise) qui caractérise la situation contemporaine. En essayant de situer la politique étrangère américaine par rapport à une logique ou un ordre quelconque, la stratégie du président Clinton rencontre une difficulté majeure: «The problem is that in this post-cold war period,

53 Voir J. Petras, «Afflux de capitaux étrangers, appauvrissement des citoyens. Investissements pervers en Amérique latine», Le Monde diplomatique, janvier 1994.

54 C. De Brie, «Perversion de la démocratie en Europe. Feu sur l'État-providence», Le Monde diplomatique, janvier 1994; aussi C. Julien, «La démocratie blessée par les injustices du système économique», Le Monde diplomatique, juin 1991.

55 J.-Y. Potel, «Aventureuse privatisation de l'économie. L'Europe de l'Est en quête de propriétaires», Le Monde diplomatique, juin 1991.

56 René Dumont, Cette guerre nous déshonore, Paris, Seuil, 1992. 
the lines just aren't as clear as they were before ${ }^{57}$ ». Le président Clinton faisait sûrement allusion au fait qu'à la suite de la disparition du spectre du communisme, la logique ou le cadre de référence que ce régime a inspiré n'étant plus légitime, les «lignes» géopolitiques du monde sont à retracer de nouveau. Adam Michnik, qui semble avoir bien connu le spectre du communisme dans sa version polonaise, décrit cette nouvelle situation d'une manière assez précise:

Pendant l'état de guerre, tout était clair. Nous et eux, le bien et le mal, la grève et les charges de la police, le choix entre l'ange et le diable. L'ange persécuté, le diable exerçant le pouvoir. Aujourd'hui, le pouvoir est exercé par l'ange et il faut vivre sans le diable. Comment vivre sans le diable du communisme? ${ }^{58}$

Cette question d'Adam Michnik décrit en fait, en les requestionnant, les enjeux politiques de la nouvelle situation. Se demander comment vivre sans le spectre du communisme signifie que l'«ange», «l'idée occidentale» selon le contexte de cet article, est au centre de la question et qu'elle s'avère beaucoup plus problématique qu'elle ne nous le laissait croire durant la grande période de peur qui a marqué la guerre froide.

Autrement dit, à défaut d'une nouvelle logique qui comblerait le vide créé par la disparition du spectre du communisme, les promoteurs de «l'idée occidentale» parlent d'un nouvel ordre mondial qui reste toujours à définir. De ce point de vue, la nouvelle situation se présente comme une sorte de no man's land à conquérir et à ordonner. En dépit de tous les «posties» qui prétendent avoir dépassé, à travers l'«écriture» ou la pensée, cette nouvelle situation (en dominant et en uniformisant les espaces discursifs), il reste en fait à repenser ces espaces et à élargir d'autres perspectives. C'est ce à quoi renvoie l'idée de «deuxième révolution» d'Adam Michnik, dans le contexte des pays de l'Europe de l'Est.

Si ce nouvel ordre mondial a encore quelque sens, celui-ci se trouve lié à la question concernant cette nouvelle situation. En ce qui regarde la question du Tiers-Monde, la nouveauté de la situation consiste dans le fait qu'on s'aperçoive que les vieux problèmes posés par les conditions qui ont généré le Tiers-Monde ont été seulement déplacés et relocalisés. À la suite de la dépolarisation idéologique de la planète, les images des différents mondes ont repris leur vrai visage et leur complexité que cachait le spectre du communisme. S'il s'est produit un changement dans cette relocalisation, ce serait en ce sens que ces vieux problèmes ont été exacerbés par le triomphe de «l'idée occidentale», donc par la modernisation mondiale de l'économique. Par conséquent, malgré ce climat triomphaliste et malgré les images de nouveauté et d'originalité créées par le nouvel ordre mondial, il s'agit plutôt en réalité de la reformulation de ces problèmes. Dans ce sens, la nouvelle situation se présente donc comme un défi.

57 Cité par B. W. Nelan. Voir «Mission to Europe», Time, 17 janvier 1994, p. 17.

58 A. Michnik, ouvrage cité, p. 57. 


\section{L'héritage de Zapata}

Paradoxalement, la conclusion qu'on peut tirer de la crise du Chiapas, telle quelle s'est déroulée jusqu'à maintenant, s'articule autour de la question de la modernisation. L'entrée en vigueur de l'ALÉNA, considéré par le gouvernement mexicain comme le couronnement de son projet de modernisation, est l'événement qui a déclenché la rébellion des paysans indiens du Chiapas. Et, jusqu'à ce jour, ce sont eux qui sont en train de moderniser l'image du Mexique selon une orientation beaucoup plus significative que celle, étroite, d'une modernisation économique se limitant aux secteurs privilégiés.

En dépit des préjugés maintenus jusqu'ici par les «gens de raison ${ }^{59}$ », les paysans indiens du Chiapas, eux, sont en train de moderniser le Mexique en ouvrant de nouveaux espaces politiques à travers l'autre image du Mexique projetée dans les mass media mondiaux. Ils contribuent à moderniser le Mexique en montrant que la crise du Chiapas est au centre de la question de l'inexistence d'un espace politique pluraliste et tolérant. Par les débats que cette crise a soulevés, l'espace politique ouvert par l'Armée zapatiste est en train de modifier sérieusement le scénario de la politique mexicaine en cette année électorale ${ }^{60}$. En considérant les enjeux politiques de la polémique provoquée dans les milieux de l'intelligentsia mexicaine, on s'aperçoit que la crise du Chiapas est en train d'actualiser aussi leurs débats.

Si le soulèvement des paysans indiens a lancé de sérieux défis politiques au gouvernement de Salinas de Gortari, en tant qu'Armée zapatiste, eux aussi se voient confrontés à des défis politiques, mais d'un autre genre. Le défi fondamental pour l'Armée zapatiste se trouve lié au mythe de l' «étranger». Dès le début de la crise, le gouvernement mexicain a essayé, comme par le passé lors des mouvements de guérilla qui ont surgi au Mexique dans les années soixante-dix (ignorés à l'extérieur du pays), de stigmatiser l'Armée zapatiste en la soupçonnant d'être liée aux intérêts étrangers. Il a tenté ainsi de l'isoler, avec le consentement tacite de la population, en faisant appel à la stabilité sociale tant exigée par la modernisation en cours.

Le problème principal qu'ont affronté au Mexique et en Amérique latine tant la gauche en général (dite marxiste) que les mouvements armés a contribué à alimenter un tel mythe. Ce problème est celui de l'adaptation des idées politiques «étrangères» (marxistes, léninistes, maoïstes, par exemple) à la réalité sociale,

59 «Gentes de razón», c'est par cette appellation - fort significative, par ailleurs que les Indiens se réfèrent encore aujourd'hui aux Mexicains. Voir l'article de Miguel Sabido, «Unas palabras a Elena, Krauze y Monsivais», La Jornada, Mexico, 10 janvier 1994.

60 Voir l'article de C. Acosta Córdova dans la revue Proceso, Mexico, no 897, 10 janvier 1994, p. 20. 
culturelle et historique d'une communauté, du Mexique dans le cas qui nous occupe. Selon Michaël Lowy:

Un des principaux problèmes qu'a dû affronter le marxisme en Amérique latine a été en effet celui de la définition du caractère de la révolution dans le continent; définition qui était à la fois le résultat d'une certaine analyse des formations sociales latino-américaines ${ }^{61}$.

Pour sa part, l'Armée zapatiste souligne elle-même ne pas suivre les modèles des guérillas précédentes ${ }^{62}$. Son idéologie et son programme politiques s'insèrent dans la problématique mexicaine, selon ses conditions socio-historiques. Le langage employé et la revalorisation de l'héritage de Zapata corroborent cette compréhension historique. L'écrivain mexicain Carlos Fuentes remarque cette authenticité historique. À l'encontre des déclarations du Prix Nobel de littérature Octavio Paz, qui reproche «aux intellectuels [mexicains] favorables aux zapatistes de ne pas avoir tiré des leçons de la chute du mur de Berlin», Carlos Fuentes, «en revanche, affirme qu'il s'agit de "la première rébellion post-communiste"» en donnant comme exemple «"le langage beaucoup plus frais et neuf" qui a remplacé le langage pétrifié et dogmatique ${ }^{63}$ ». Finalement, ce qui ressort de cette authenticité historique, c'est l'héritage de Zapata.

\author{
Carlos AGUILAR \\ Doctorat en littérature comparée \\ Université de Montréal
}

\title{
Résumé
}

Dans cet article, je propose d'explorer la question concernant la crise politique et sociale déclenchée au Mexique par le soulèvement inattendu des paysans indiens de l'Armée zapatiste du Chiapas. En exposant une autre image du Mexique dans un moment crucial de son histoire, l'Armée zapatiste réactualise les contrastes et les contradictions qui caractérisent ce pays. En alternant et en nuançant les questions de la représentation et des faits historico-politiques soulevés par cette crise, il s'agit d'ouvrir d'autres espaces de discussion en replaçant de telles questions dans un contexte plus large. Le but ultime de cet article est donc de cerner de nouveaux espaces de lisibilité pour reconnaître la question posée par les paysans indiens du Chiapas malgré le climat peu réceptif dégagé par le nouvel ordre mondial.

61 M. Lowy, Le marxisme en Amérique latine de 1909 à nos jours, Paris, François Maspero, 1980, p.7.

62 «¿de qué nos van a perdonar?», lettre de l'Armée zapatiste publiée dans le quotidien La Jornada, Mexico, 21 janvier 1994.

63 Cité par B. de la Grange, Le Devoir, article cité. 
Mots-clés: Mexique, zapatiste, crise, Indiens, mondialisation, image.

\section{Summary}

This article discusses the social and political crisis in Mexico resulting from the uprising of Chiapas peasants of Indian ancestry. By revealing a different image of Mexico at a crucial moment in its history, the Zapatista army re-actualizes the contrast and contradictions characterizing this country. By alternating and comparing issues related to the representation and the historico-political facts raised by this crisis, I open new spaces of discussion and relocate such issues in a broader context. The ultimate goal and challenge of this article is thus one of finding new spaces of legibility, thereby allowing for the recognition of the issues raised by the Chiapas uprising despite the unreceptive climate created by the new world order.

Key-words: Mexico, Zapatista, crisis, Indians, globalization, images.

\section{Resumen}

En este artículo me propongo explorar la cuestión relacionada con la crisis política y social suscitada en México por la inesperada rebelión de los indioscampesinos de Chiapas. Al mostrar otra imagen de México en un momento crucial de su historia, el Ejército zapatista reactualiza los contrastes y las contradicciones que caracterizan este país. Alternando y contrastando las cuestiones de la representación y de los hechos históricos y políticos provocados por tal crisis, intento abrir otros espacios de discusión resituando tales cuestiones en un contexto más amplio. El fin último (y el desafío) de este artículo consiste por lo tanto en encontrar nuevos espacios de legibilidad para así reconocer la cuestión planteada por los campesinos-indios de Chiapas a pesar de la atmósfera poco receptiva que se desprende dell nuevo orden mundial.

Palabras claves: Mexico, zapatista, crisis, Indios, mundialización, imagen. 